\title{
Validation d'un banc d'étalonnage primaire en microdébitmétrie liquide et application aux dispositifs médicaux de perfusion
}

\author{
Validation of a liquid micro-flow primary \\ calibration bench and application to drug \\ delivery devices
}

\section{Florestan OGHEARD}

LNE-CETIAT (Centre Technique des Industries Aérauliques et Thermiques), 25 Avenue des Arts, 69603 Villeurbanne Cedex, France, florestan.ogheard@ cetiat.fr.

\section{Résumé}

Antérieurement à 2012, la traçabilité des mesures de micro- et nanodébits de liquides (de l'ordre de $1 \mathrm{~mL} \cdot \mathrm{h}^{-1}$ et en deçà) n'était pas assurée en raison de l'absence de références métrologiques. Pour des applications liées au secteur de la santé, telle que l'administration de médicaments par perfusion, l'incertitude sur le débit réel d'injection aux patients peut avoir des conséquences dramatiques, voire létales. La qualité du dosage volumétrique est de fait un paramètre critique très important à maîtriser. La connaissance précise du volume administré et la stabilité du débit d'injection sont cruciales, particulièrement pour les médicaments qui requièrent une très faible concentration sanguine pour des raisons de toxicité, tels que les médicaments vasoactifs ou anesthésiants. Pour répondre à ce besoin, les laboratoires nationaux de métrologie LNE-CETIAT, DTI, IPQ, METAS et VSL ont développé, dans le cadre du projet européen de recherche "EMRP/HLT07 MeDD - Metrology for Drug Delivery", des installations d'étalonnage couvrant une gamme de débits de liquide allant de $600 \mathrm{~nL} \cdot \mathrm{h}^{-1}$ à $600 \mathrm{~mL} \cdot \mathrm{h}^{-1}$. Cet article présente le travail effectué au LNE-CETIAT dans le cadre de ce projet, consistant à qualifier un banc de référence pour des débits de liquide compris entre $1 \mathrm{~mL} \cdot \mathrm{h}^{-1}$ et $10 \mathrm{~L} \cdot \mathrm{h}^{-1}$ et à étudier l'influence de la température sur les performances métrologiques des microdébitmètres et dispositifs médicaux de perfusion.

MOTS CLÉS : DISPOSITIFS MÉDICAUX DE PERFUSION, MICRODÉBIT LIQUIDE, ÉTALONNAGE GRAVIMÉTRIQUE, ÉTALON PRIMAIRE, MÉTROLOGIE POUR L'ADMINISTRATION DE MÉDICAMENTS.

\footnotetext{
Abstract

Until 2012, micro- and nano-flow rate measurements traceability was a problem, due to the lack of references at and below $1 \mathrm{~mL} \cdot \mathrm{h}^{-1}$ For drug delivery by infusion, uncertainty on real delivered flow rate
}

can cause injuries or even death. Quality of volumetric dosing has to be closely controlled. Exact delivered volume and stability are critical parameters, particularly for drugs requiring low blood concentration for toxicity reasons, such as vasoactive and anesthetics. To meet this need, the national metrology laboratories LNE-CETIAT, DTI, IPQ, METAS and VSL have developed, in the scope of the European Research Project in Metrology "EMRP/HLTO7 MeDD - Metrology for Drug Delivery", calibration benches for flow rates from $600 \mathrm{~mL} \cdot \mathrm{h}^{-1}$ down to $600 \mathrm{~nL} \cdot \mathrm{h}^{-1}$. This article presents the work done at LNE-CETIAT in the scope of this project, including qualification of a calibration facility for flow rates from $1 \mathrm{~mL} \cdot \mathrm{h}^{-1}$ to $10 \mathrm{~L} \cdot \mathrm{h}^{-1}$, and temperature assessment of microflowmeters and drug delivery devices.

KEY WORDS: DRUG DELIVERY DEVICES, LIQUID MICRO-FLOW, GRAVIMETRIC CALIBRATION, PRIMARY STANDARD, METROLOGY FOR DRUG DELIVERY.

\section{Introduction}

Les professionnels du milieu médical admettent que les risques liés à l'utilisation d'un dispositif de perfusion sont sous-estimés [1], particulièrement aux très faibles débits (de l'ordre de $1 \mathrm{~mL} \cdot \mathrm{h}^{-1}$ ). Ces risques peuvent conduire à une mauvaise efficacité des traitements. L'usage grandissant des perfusions multi-pompes nécessite un meilleur contrôle des concentrations en médicament, et donc du débit d'injection. L'influence des caractéristiques du dispositif de délivrance de médicament (délai de démarrage, compliance ${ }^{1}$, stabilité du débit)

\footnotetext{
${ }^{1}$ La compliance représente «l'élasticité » du système de perfusion; voir paragraphe 4 de l'article.
} 
et l'effet des conditions d'utilisation sur la concentration sont mal connus. En général, le paramètre le plus important, lors de l'administration d'un médicament par perfusion, est le volume (ou la masse) délivré au patient. Cependant, un nombre significatif de médicaments nécessite de connaître le débit réel d'administration du médicament au patient afin de garantir l'effet thérapeutique désiré.

Les fournisseurs de dispositifs médicaux de perfusion annoncent la génération de gammes très étendues de débits (de $60 \mathrm{~nL} \cdot \mathrm{h}^{-1}$ jusqu'à $10 \mathrm{~L} \cdot \mathrm{h}^{-1}$ ), avec des incertitudes associées de l'ordre de quelques pourcents, mais ces incertitudes ne sont pas validées. En général, toutes les composantes d'incertitude connues ne sont pas prises en compte par les constructeurs ou les utilisateurs. Dans le cas de dispositifs médicaux de perfusion, l'incertitude résultant de l'association de plusieurs dispositifs et de divers accessoires est également mal connue.

Pour répondre à ce besoin de traçabilité des mesures des très faibles débits, les laboratoires nationaux de métrologie LNE-CETIAT (France), DTI (Danemark), IPQ (Portugal), METAS (Suisse) et VSL (Pays-Bas) se sont engagés dans le projet de recherche collaboratif (JRP) «HLT07 MeDD - Metrology for Drug Delivery »[2] mené dans le cadre de l'appel de 2011 du programme européen de recherche en métrologie (EMRP) d'Euramet dans la thématique Santé. Ce projet s'est déroulé sur trois années (de juin 2012 à juin 2015) et a eu pour objectif principal de développer, au sein de ces instituts européens, des moyens d'étalonnage traçables pour la mesure de faibles débits; l'objectif à moyen terme étant de développer des outils pour améliorer la fiabilité de la délivrance de médicament, notamment à l'aide de perfusions multi-pompes et pour d'autres applications à très faibles débits. Le projet avait été construit en quatre lots de tâches :

- Lot $n^{\circ} 1$ : développement de la première chaîne de traçabilité pour des débits de liquide de $600 \mathrm{~nL} \cdot \mathrm{h}^{-1}$ à $600 \mathrm{~mL} \cdot \mathrm{h}^{-1}$, avec une incertitude cible de $0,5 \%$;

- Lot $\mathrm{n}^{\circ} 2$ : évaluation métrologique de microdébitmètres commercialisés ;

- Lot $n^{\circ} 3$ : évaluation métrologique de dispositifs médicaux de perfusion associés à divers accessoires ;

- Lot $\mathrm{n}^{\circ} 4$ : diffusion des résultats obtenus, via différents types de publication, à destination des communautés scientifiques et médicales.

Dans ce JRP, le LNE-CETIAT a orienté sa participation autour de la validation d'un nouveau banc primaire, qu'il avait déjà développé et inauguré en 2012, et de la caractérisation métrologique de microdébitmètres et de dispositifs médicaux de perfusion, notamment en fonction de la température du fluide.

Cet article présente les moyens déjà développés par le LNE-CETIAT, un banc d'étalonnage de référence pour les mesures de microdébits de liquide, les résultats de la comparaisons réalisée dans le cadre du JRP MeDD pour valider cette référence et l'application du banc à l'étalonnage des microdébitmètres utilisés dans les dispositifs médicaux de perfusion.

\section{Validation d'une référence nationale en microdébitmétrie liquide}

Le premier lot de tâches a permis de développer et de valider, au sein des laboratoires partenaires, l'utilisation de bancs d'étalonnage primaires pour couvrir des débits de liquide de $600 \mathrm{~nL} \cdot \mathrm{h}^{-1}$ jusqu'à $10 \mathrm{~L} \cdot \mathrm{h}^{-1}$ et permettre l'étalonnage des dispositifs médicaux de perfusion. Le principe de fonctionnement général des bancs développés repose sur une méthode gravimétrique. Il met en œuvre un générateur de débit, des conduites permettant le raccordement du débitmètre à étalonner, et une partie «mesure » composée d'une balance de précision sur laquelle est disposé un réservoir de pesée collectant le liquide. Afin de maîtriser les paramètres d'influences liés aux propriétés physicochimiques du liquide en écoulement, notamment en réalisant le traitement spécifique décrit ciaprès, l'eau a été choisie comme liquide d'étalonnage pour la référence nationale développée au LNE-CETIAT. Par ailleurs, dans le cadre du projet MeDD, des essais spécifiques utilisant un liquide autre que l'eau, ont été réalisés par les laboratoires METAS et IPQ. Ces essais spécifiques ont permis de quantifier l'influence des propriétés physicochimiques du liquide en circulation sur les performances métrologiques de microdébitmètres et dispositifs médicaux de perfusion [3,4].

\subsection{Description de l'installation du LNE-CETIAT}

Afin d'assurer la meilleure stabilité possible du débit généré, la mise en œuvre d'un banc d'étalonnage en microdébitmétrie liquide nécessite le contrôle des paramètres environnementaux. L'eau nécessaire à l'étalonnage est dans un premier temps déminéralisée puis dégazée à l'azote afin d'éviter le dépôt de minéraux et la formation de bulles d'air dans le circuit. Le dégazage est réalisé en deux étapes : la première consiste à faire circuler de l'azote par bullage dans un réservoir rempli d'eau déminéralisée, la seconde voit l'eau, ainsi pré-dégazée, circuler en boucle fermée dans un réservoir fermé et dont on a évacué l'air présent dans le volume restant grâce à une pompe à vide. Enfin, l'eau est thermalisée à la température de consigne désirée, de $10{ }^{\circ} \mathrm{C}$ à $50^{\circ} \mathrm{C}$ (fig. 1).

L'eau ainsi préparée est ensuite transférée au réservoir permettant la génération du débit. Ce réservoir est maintenu à une pression constante, pouvant être ajustée de 0,3 bar à 10 bar $\left(1\right.$ bar $\left.=10^{5} \mathrm{~Pa}\right)$, grâce à un soufflet métallique pressurisé à l'azote afin d'éviter l'introduction d'air dans le circuit en cas de microfuite du soufflet.

L'ensemble générateur de débit est placé dans une enceinte climatique permettant d'asservir la température d'eau entre $10^{\circ} \mathrm{C}$ et $50^{\circ} \mathrm{C}$ (fig. 2).

L'appareil en étalonnage est installé dans une deuxième enceinte climatique. Le débit généré est transmis du générateur vers l'appareil en étalonnage, via un capillaire calorifugé. 


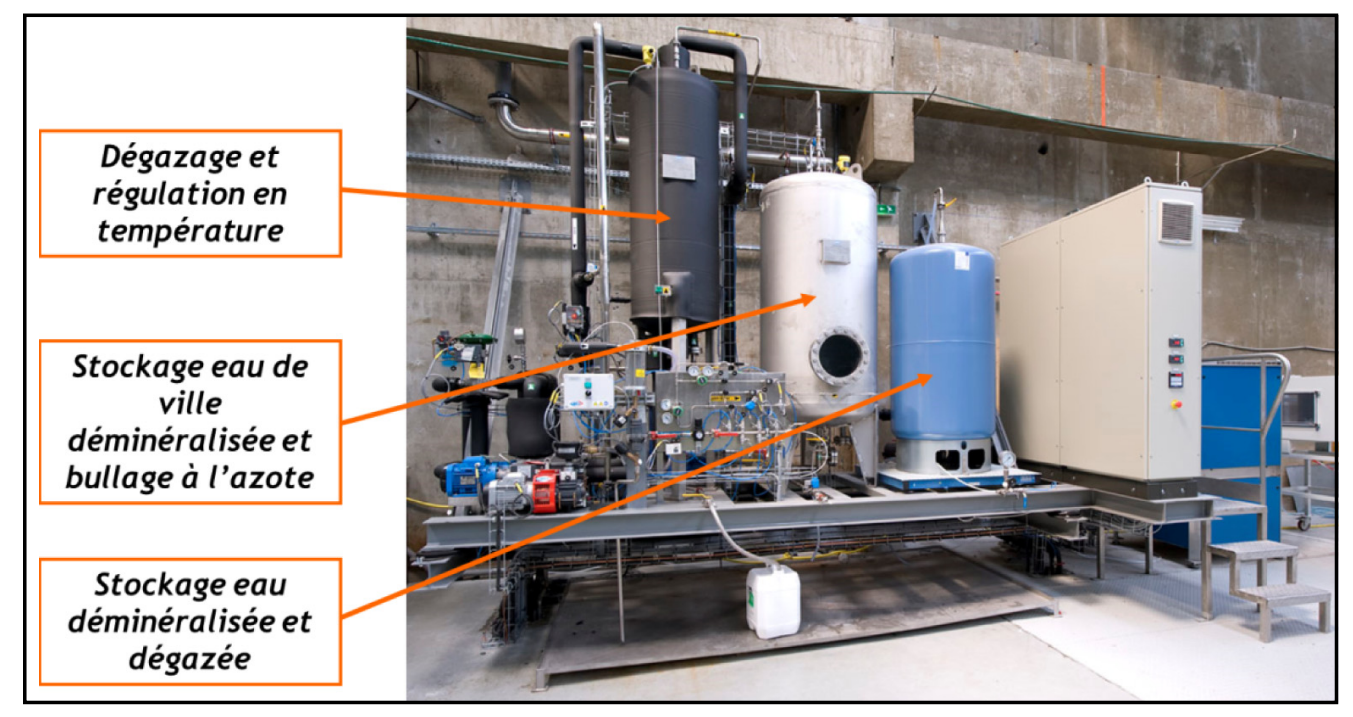

Fig. 1. - Photographie de l'ensemble de préparation de l'eau servant à l'étalonnage.

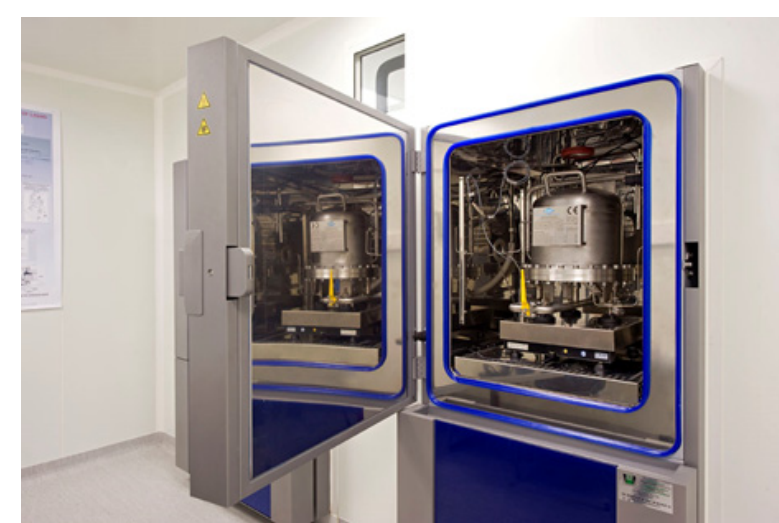

Fig. 2. - Photographie de l'ensemble générateur de débit.

L'eau en circulation passe alors dans la partie «mesure » de l'installation. Cette partie située dans une salle propre dédiée (nommée «enceinte laboratoire » dans la fig. 3) permet d'assurer la stabilité des conditions de mesure (pression, température, humidité, vibrations, pollution particulaire). Le conditionnement en température du liquide est assuré par trois échangeurs (échangeur $\mathrm{n}^{\circ} 1$, échangeur $n^{\circ} 2$ et bain thermostaté, voir fig. 3) permettant d'obtenir une stabilité de $\pm 0,01{ }^{\circ} \mathrm{C}$ à $20^{\circ} \mathrm{C}$ dans la section du circuit présentant la plus forte perte de charge, et assure donc une stabilité du débit optimale.

Un jeu de 11 capillaires, montés en parallèle et associés à des électrovannes, permet de sélectionner la gamme de pertes de charge nécessaire à la réalisation des débits correspondants. En effet, le débit généré est défini grâce au choix du couple (pression dans le réservoir générateur; diamètre du capillaire). Le fluide (l'eau) s'écoule, avec le débit ainsi établi, dans le système de pesée constitué de quatre balances, chacune dimensionnée pour une gamme de débits distincte, assurant ainsi une homogénéité des temps de mesure et des incertitudes sur la mesure du débit.

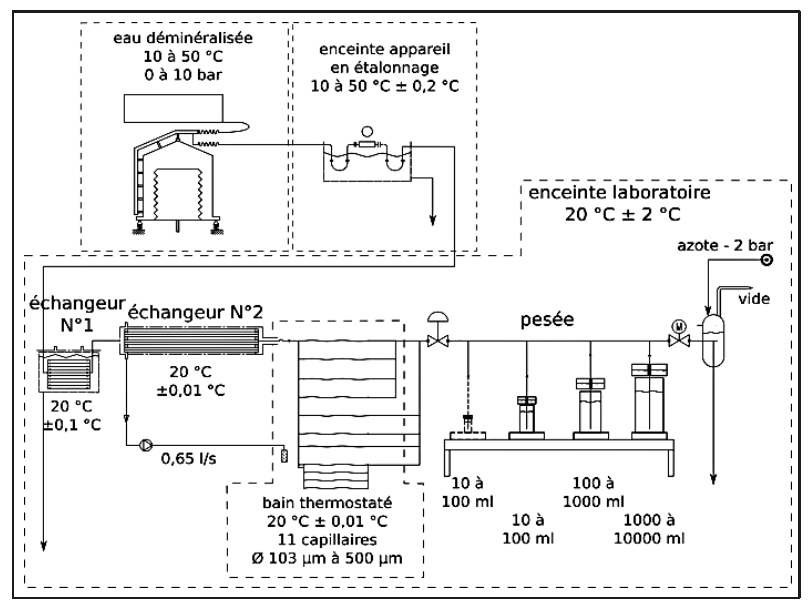

Fig. 3. - Schéma simplifié du banc d'étalonnage.

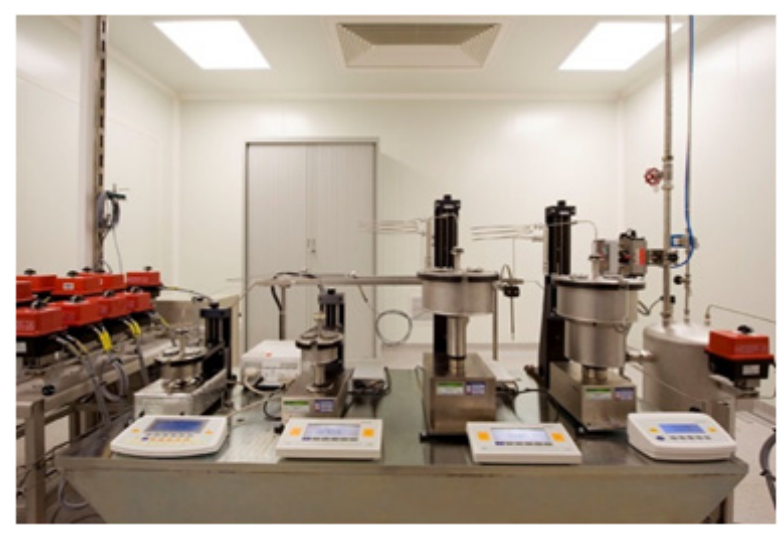

Fig. 4. - Photographie du système de pesée.

Le système de pesée du fluide permettant la détermination du débit de référence a été installé sur un marbre assurant une coupure à $3 \mathrm{~Hz}$ des vibrations environnantes (fig. 4).

Pour chaque balance, le fluide s'écoule, avec le débit généré, dans un réservoir en verre associé à un saturateur 


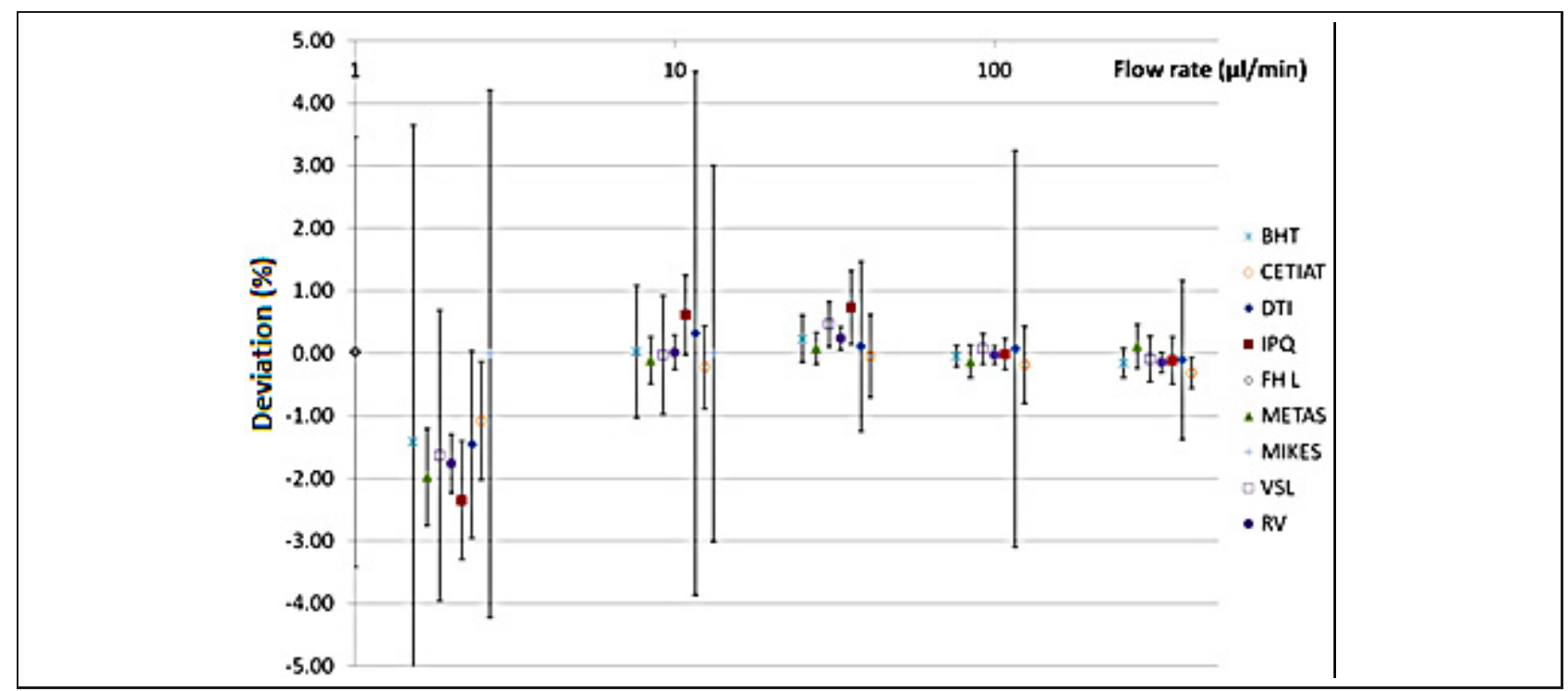

Fig. 5. - Résultats de la comparaison interlaboratoire pour le pousse-seringue.

limitant l'évaporation. Le capillaire amenant le fluide dans le réservoir de pesée est doté d'orifices de sortie conçus pour éliminer l'impact du jet d'eau sur la mesure de masse. L'ensemble est dimensionné pour limiter les effets de variation de tension superficielle et de poussée d'Archimède pendant la mesure.

\subsection{Mesure du débit par une méthode gravimétrique}

Une méthode gravimétrique est mise en œuvre grâce à l'association des balances à un système de mesure du temps. La pesée commence à l'ouverture d'une vanne rapide, placée en amont du système de pesée, qui déclenche simultanément l'enregistrement des mesures par la balance et le système de mesure du temps. La fin de la mesure est déclenchée à l'obtention de la consigne de masse prédéterminée (de $0,5 \mathrm{~g}$ à $500 \mathrm{~g}$ selon la balance) : la vanne se ferme et déclenche l'arrêt de l'enregistrement du couple (masse, $m$; temps, $t$ ). Le débit massique est obtenu en calculant le rapport entre ces deux grandeurs mesurées, $\frac{\Delta m}{\Delta t}=\frac{m_{\mathrm{f}}-m_{\mathrm{i}}}{t_{\mathrm{f}}-t_{\mathrm{i}}}$, avec $\Delta m$ la différence entre les masses pesées à l'ouverture (indice i) et à la fermeture (indice f) de la vanne rapide, et $\Delta t$ le temps écoulé mesuré. L'ensemble du processus d'étalonnage est géré par un automate contrôlé par un logiciel de supervision dédié, et installé sur un poste opérateur à proximité de la salle propre.

La référence nationale développée au LNE-CETIAT pour les mesures de débits de $1 \mathrm{~g} \cdot \mathrm{h}^{-1}$ à $10 \mathrm{~kg} \cdot \mathrm{h}^{1}$ a été inaugurée en 2012 et ses incertitudes relatives élargies $(k=2)$ ont été évaluées de $0,1 \%$ à $0,6 \%$.

\subsection{Comparaison interlaboratoire}

Dans le cadre du lot de tâches $\mathrm{n}^{\circ} 1$ du JRP MeDD, les laboratoires IPQ, METAS, LNE-CETIAT, VSL et DTI ont développé des bancs d'étalonnage couvrant globalement des débits de liquide allant de $600 \mathrm{~nL} \cdot \mathrm{h}^{-1}$ à $10 \mathrm{~L} \cdot \mathrm{h}^{-1}$. Le principe général d'une mesure gravimétrique a été appliqué par tous ces laboratoires pour la mesure du débit de référence, avec quelques variantes expliquant les différentes incertitudes relatives proposées par les laboratoires [5].

Ces bancs d'étalonnage (et les incertitudes correspondantes) ont été validées par une comparaison interlaboratoire organisée dans le cadre du JRP [6] pour des débits allant de $120 \mu \mathrm{L} \cdot \mathrm{h}^{-1}$ à $600 \mathrm{~mL} \cdot \mathrm{h}^{-1}$. Deux étalons de transfert ont été utilisés : un pousse-seringue et un débitmètre massique à effet Coriolis. Le constructeur de microdébitmètres Bronkhorst High-Tech, l'institut national de métrologie MIKES (Finlande) et l'université de Lübeck (Allemagne) ont également participé à cette comparaison. Les résultats obtenus (fig. 5 et 6) sont cohérents entre eux, à l'exception de ceux mesurés par le débitmètre au débit maximal. Cette incohérence a été expliquée a posteriori par des corrections non systématiquement appliquées. Les figures 5 et 6 présentent les erreurs de justesse (et les incertitudes correspondantes) déterminées par comparaison du débit mesuré par méthode gravimétrique, au débit mesuré par l'étalon de transfert. Sur ces figures, « RV » (Reference Value) symbolise la valeur de référence obtenue par combinaison des résultats de l'ensemble des participants.

\section{Caractérisation de l'influence de la température sur les performances métrologiques de microdébitmètres liquide}

L'étalonnage de dispositifs médicaux de perfusion peut être réalisé par comparaison à un microdébitmètre, étalon de transfert raccordé à l'une des références nationales développées. Afin d'évaluer des microdébitmètres pouvant constituer des étalons de transfert, les trois instruments suivants ont été caractérisés :

- un débitmètre massique à effet Coriolis (Bronkhorst M12); 


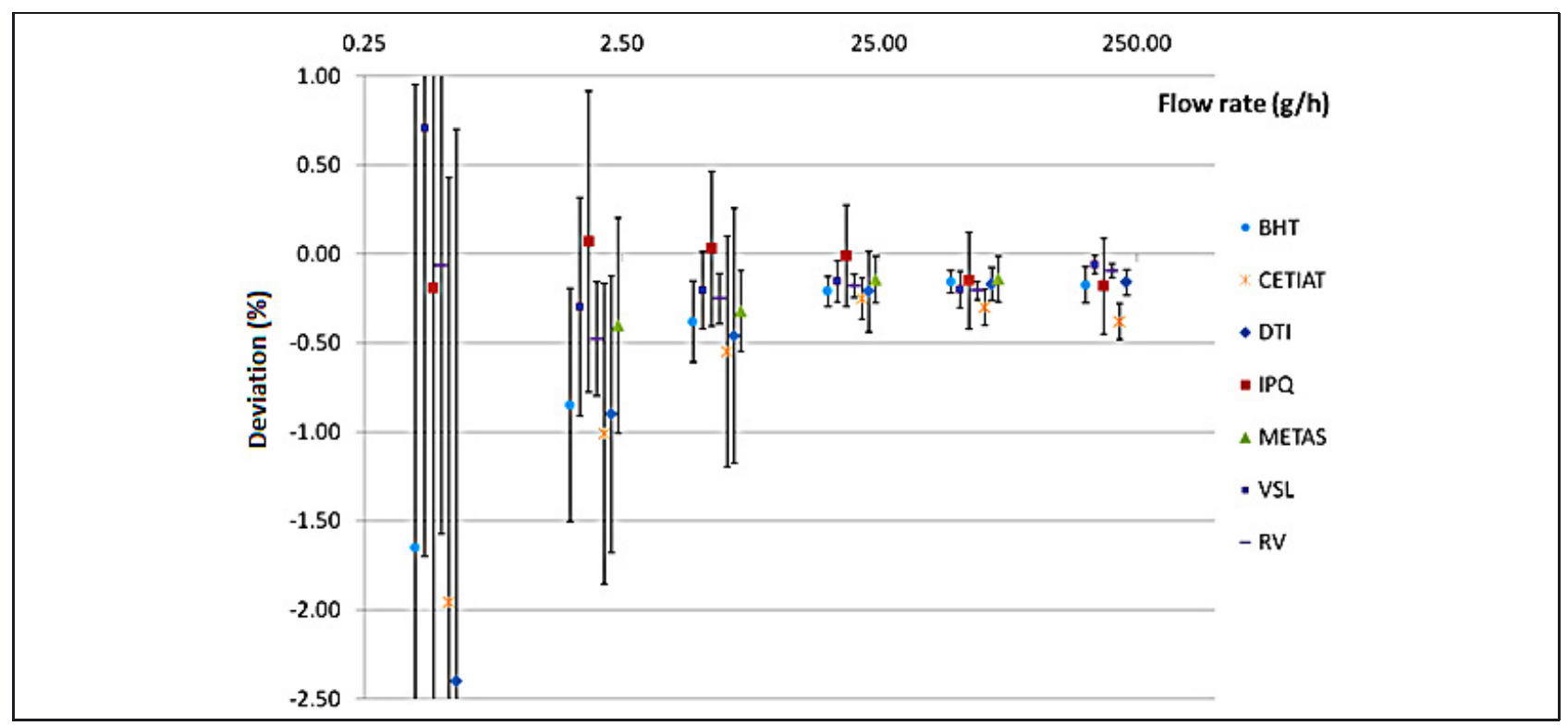

Fig. 6. - Résultats de la comparaison interlaboratoire pour le débitmètre.

\section{Erreur relative moyenne - Bronkhorst M12}

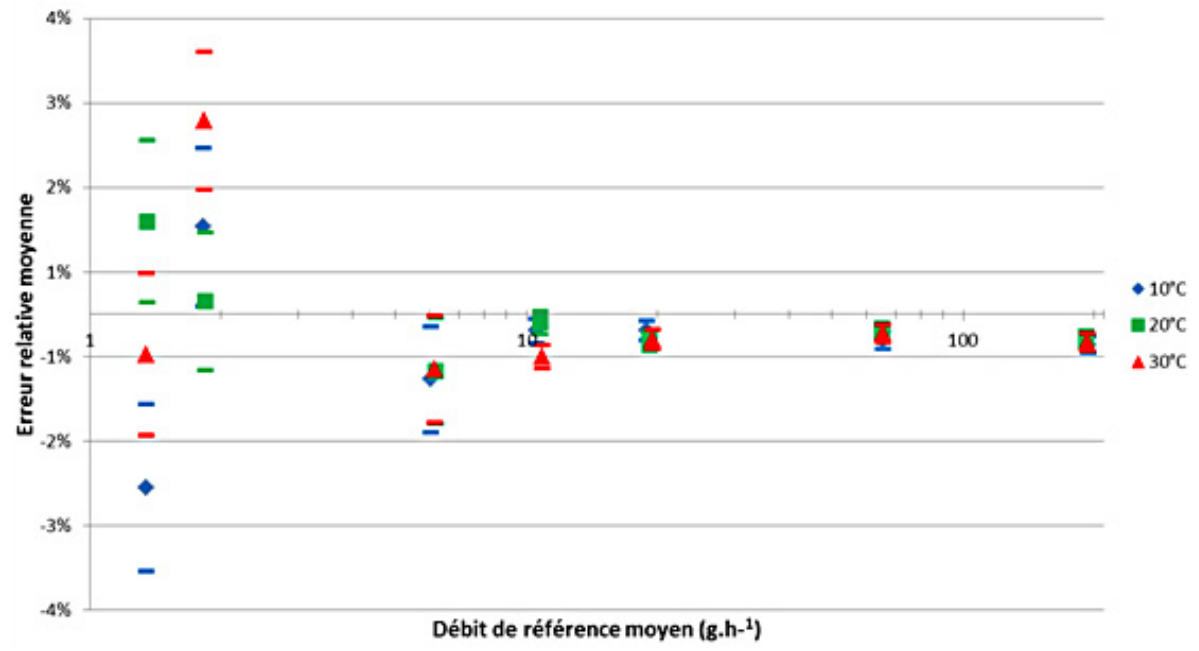

Fig. 7. - Résultats de l'étalonnage du débitmètre Bronkhorst M12 pour trois températures de l'eau.

- un débitmètre à différentiel de pression (Alicat Scientific L0.5CCM-D);

- un débitmètre thermique (Sensirion SLI-0430).

Les performances métrologiques (erreur de justesse et fidélité) de ces débitmètres ont été déterminées à l'aide du banc de référence primaire développé au CETIAT, pour trois valeurs de température de l'eau $\left(10{ }^{\circ} \mathrm{C}, 20{ }^{\circ} \mathrm{C}\right.$ et $30^{\circ} \mathrm{C}$ ) afin d'étudier l'influence de la température sur ces instruments. Les figures 7-9 présentent les résultats obtenus en termes d'erreurs de justesse relatives du débitmètre par rapport au débit mesuré par méthode gravimétrique.

Les résultats obtenus montrent que la température (dans les conditions de mesure) a un effet négligeable sur la réponse du débitmètre massique à effet Coriolis et du débitmètre à différentiel de pression. Le débitmètre thermique, quant à lui, est plus sensible à la température du fluide (notamment à $10{ }^{\circ} \mathrm{C}$ ). Ceci peut être dû aux approximations du modèle intrinsèque au débitmètre compte tenu de la variation de la chaleur spécifique de l'eau en fonction de la température. En effet, la chaleur spécifique de l'eau varie 2,5 fois plus entre $10^{\circ} \mathrm{C}$ et $20^{\circ} \mathrm{C}$ qu'entre $20^{\circ} \mathrm{C}$ et $30^{\circ} \mathrm{C}$.

\section{Caractérisation de l'influence de la température sur les performances métrologiques de dispositifs médicaux de perfusion}

Les dispositifs médicaux de délivrance de médicaments sont très largement utilisés en médecine clinique. Leur principale fonction est de participer à un traitement par médicaments, à la nutrition ou à l'hydratation intraveineuse du patient, que ce soit à l'hôpital ou 


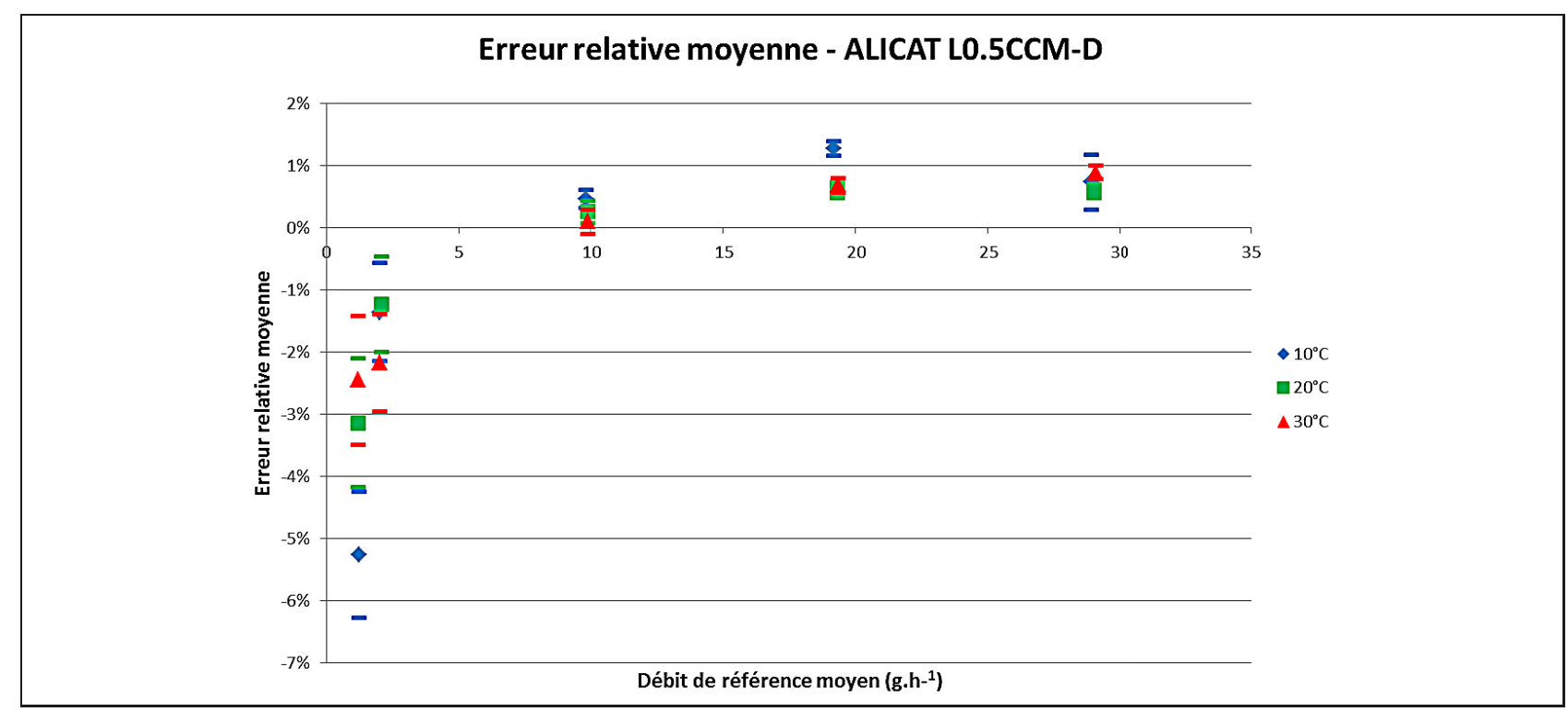

Fig. 8. - Résultats de l'étalonnage du débitmètre Alicat L0.5CCM-D pour trois températures de l'eau.

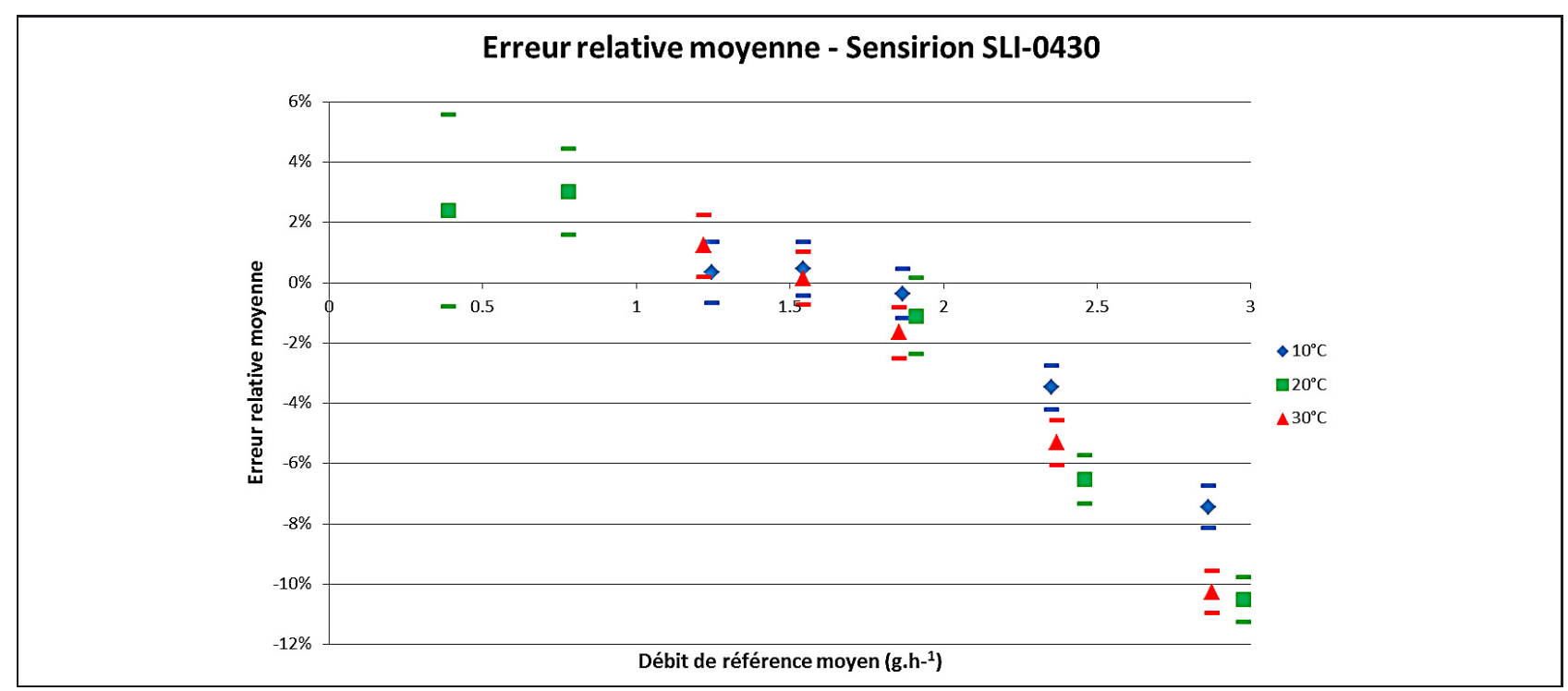

Fig. 9. - Résultats de l'étalonnage du débitmètre Sensirion SLI-0430 pour trois températures de l'eau.

au domicile de ce dernier. Plusieurs études internationales [7] montrent que l'incertitude sur le dosage de médicament par perfusion peut être non négligeable, pouvant aller jusqu'à compromettre l'efficacité du traitement délivré au patient. Dans les applications de perfusion, le dosage est contrôlé par l'ajustement du débit de la pompe et par la connaissance de la concentration du médicament dans la solution administrée au patient.

Dans le cadre de ces applications médicales, la fiabilité de ces dispositifs doit être connue et maîtrisée. Cependant, les procédures normalisées décrites dans la norme ISO60601-2-24 [8] ne prennent pas en compte les variations rencontrées lors de l'usage de ces dispositifs, que ce soit en termes d'accessoires, de configurations possibles, ou de conditions opérationnelles (nature du liquide, température ambiante, etc.). Pour les applications critiques de délivrance de médicament, il est important de connaître et de maîtriser le débit délivré par l'ensemble de perfusion (pompe, tubes et accessoires).

Les travaux du LNE-CETIAT, s'inscrivant dans cette partie du projet européen MeDD (lot de tâches $n^{\circ} 3$ ), ont porté sur la caractérisation de l'influence de la température sur les performances métrologiques du dispositif en essai. Les essais ont été réalisés sur deux types de dispositifs : un pousse-seringue et une pompe péristaltique à perfusion. Les caractéristiques qui ont été évaluées sont les suivantes :

- $t_{95 \%}$ : temps moyen mis par le dispositif pour atteindre $95 \%$ du débit de consigne, aussi appelé «temps de démarrage »;

- stabilité du dispositif : écart type sur le débit «stable» mesuré pendant une durée donnée, après le temps de démarrage ; 


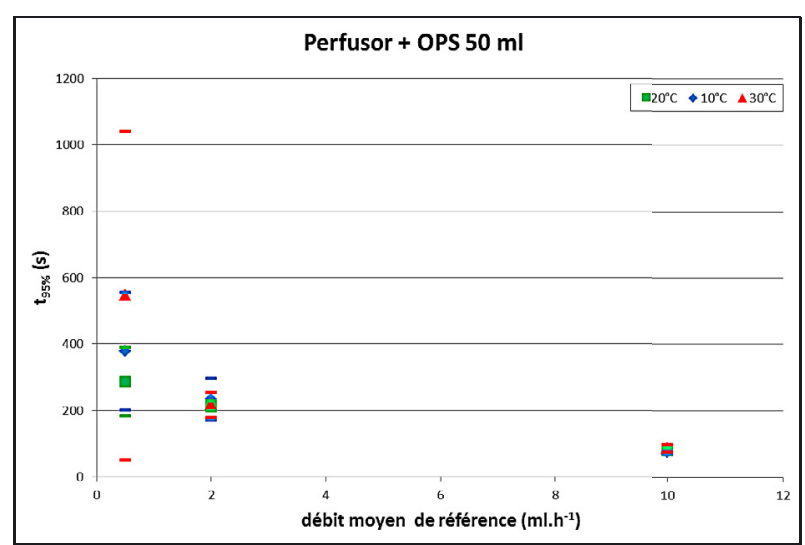

Fig. 10. - Temps de démarrage du pousse-seringue «Perfusor» associé à une seringue « $\mathrm{OPS} »$ de $50 \mathrm{~mL}$, pour des températures de $10^{\circ} \mathrm{C}, 20^{\circ} \mathrm{C}$ et $30^{\circ} \mathrm{C}$.

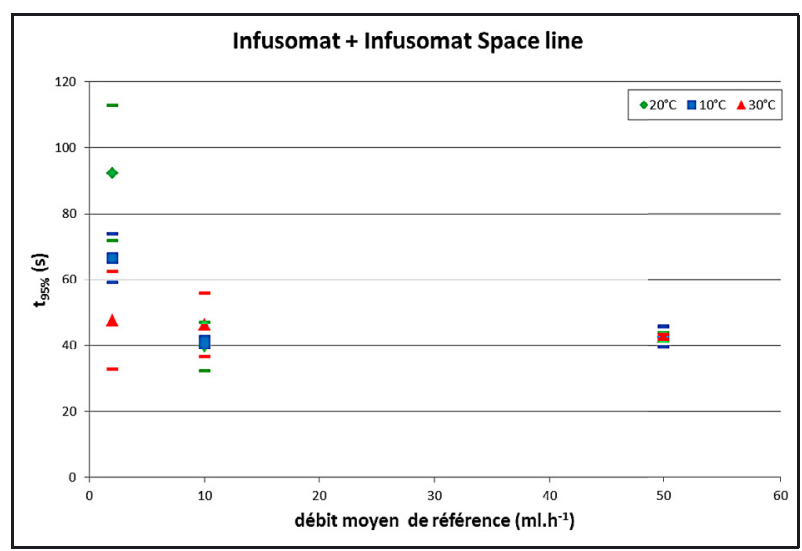

Fig. 11. - Temps de démarrage de la pompe à perfusion «Infusomat » associé à une tubulure «Infusomat Space Line », pour des températures de $10{ }^{\circ} \mathrm{C}, 20^{\circ} \mathrm{C}$ et $30^{\circ} \mathrm{C}$.

- erreur relative : erreur de justesse relative entre le débit de consigne et le débit de référence;

- compliance : rapport $C=\frac{\Delta V}{\Delta P}$ avec $\Delta V$ et $\Delta P$ les différences de volume et pression de ligne constatées entre l'occlusion volontaire du dispositif de perfusion et la détection de cette occlusion. La pression de ligne est mesurée par un capteur de pression monté à l'aval du dispositif, le volume est calculé en faisant le produit du débit stable, mesuré avant occlusion, par le temps de détection de cette occlusion. La compliance représente ainsi l'élasticité du système de perfusion.

Les figures 10 à 13 présentent les deux caractéristiques pour lesquelles les résultats les plus intéressants ont été obtenus, soit le temps de démarrage et la compliance des systèmes évalués. L'ensemble des mesures réalisées dans ce lot de tâches $\left(\mathrm{n}^{\circ} 3\right)$ est détaillé dans l'article de Bastista [4].

Concernant le délai de démarrage (fig. 10 et 11), l'étude menée au LNE-CETIAT a montré que la température (ambiante et du fluide) impacte surtout la répétabilité des mesures à faibles débits. Dans tous les cas, le délai de démarrage pour le pousse-seringue est bien

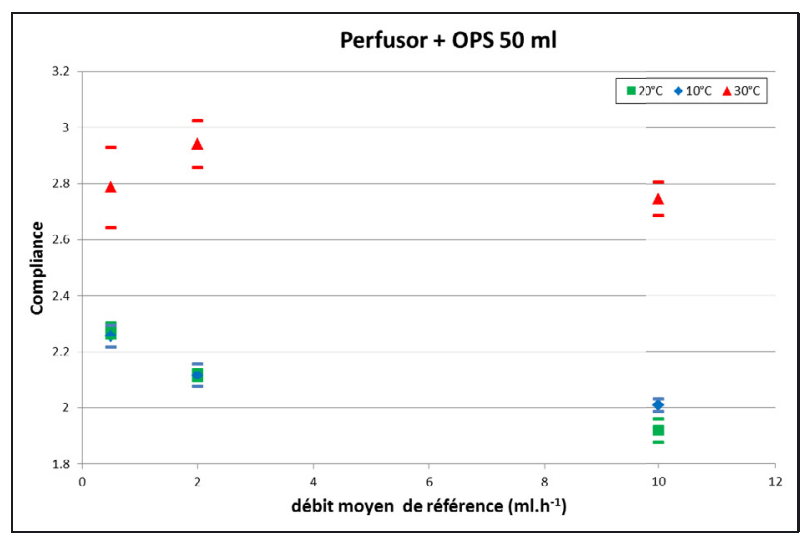

Fig. 12. - Compliance du pousse-seringue «Perfusor» associé à une seringue « $\mathrm{OPS} »$ de $50 \mathrm{~mL}$, pour des températures de $10{ }^{\circ} \mathrm{C}, 20^{\circ} \mathrm{C}$ et $30^{\circ} \mathrm{C}$.

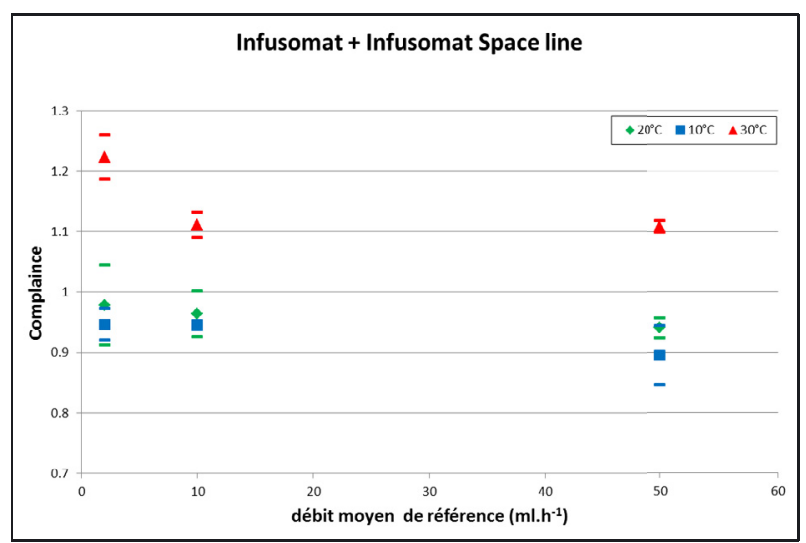

Fig. 13. - Compliance de la pompe à perfusion «Infusomat» associée à une tubulure «Infusomat Space Line », pour des températures de $10{ }^{\circ} \mathrm{C}, 20^{\circ} \mathrm{C}$ et $30^{\circ} \mathrm{C}$.

supérieur (d'un facteur 2 à 10 ) à celui mesuré pour la pompe péristaltique à perfusion. Ceci s'explique par la différence de volume occupé par le liquide en perfusion pour les deux dispositifs.

Une influence de la température du fluide est également constatée pour la compliance (fig. 12 et 13), avec un écart systématique (facteur environ égal à 1,2) entre d'une part, les résultats obtenus à $10{ }^{\circ} \mathrm{C}$ et $20^{\circ} \mathrm{C}$, et d'autre part ceux obtenus à $30^{\circ} \mathrm{C}$, quel que soit le dispositif. Cette augmentation de la compliance est due aux matériaux plastiques employés dans ce type de dispositif dont l'élasticité augmente avec la température.

\section{Conclusion}

Les activités menées au sein du projet «JRP MeDD » ont permis de valider les références nationales en microdébitmétrie liquide des partenaires, dont celle du LNECETIAT, adaptées notamment à l'étalonnage de dispositifs médicaux de perfusion. La validation du banc d'étalonnage primaire français en microdébitmétrie liquide a été réalisée grâce à une comparaison interlaboratoire pour laquelle un pousse-seringue et un microdébitmètre furent utilisés comme étalons de transfert. Ce banc 
de référence de débitmétrie a ensuite été utilisé pour étudier l'influence de la température du fluide sur les performances métrologiques de microdébitmètres et de dispositifs médicaux de perfusion. Ainsi, les caractéristiques d'intérêt pour les usages cliniques, tels que le délai de démarrage, la stabilité du débit et la compliance d'un pousse-seringue et d'une pompe à perfusion ont, pour la première fois, été déterminées avec une traçabilité métrologique.

\section{Références}

[1] SNIJDER R.A. et al., "Flow variability and its physical causes in infusion technology: a systematic review of in vitro measurement and modeling studies", Biomed. Eng.-Biomed. Tech., 60, 4, 2015, 277-300.

[2] Lucas P. et al., "Metrology for Drug Delivery", Projet EURAMET-EMPIR HLT07 MeDD, 2012-2015, www. drugmetrology.com.

Article reçu le 14 décembre 2015, version révisée reçue le 18 juillet 2016.
[3] Bissig H. et al., "Flow meter assessment (D2.3.3, D2.3.6, D2.3.7)", rapport publié sur le site www.drugmetrology. com (2015).

[4] Batista E. et al., "Assessment of drug delivery devices", Biomed. Eng.-Biomed. Tech., 60, 4, 2015, 347-357.

[5] Bissig H., Petter H.T., Lucas P., Batista E., Filipe E., Almeida N., Ribeiro L.F., Gala J., Martins R., SAVANiER B., OGHEARD F., NiEMANN A.K., LÖTTERS J. et SPARREBOom W., "Primary Standards for Flow Rates from $100 \mathrm{nl} \cdot \mathrm{min}^{-1}$ to $1 \mathrm{ml} \cdot \mathrm{min}^{-1}$ - Gravimetric Principle", Biomed. Eng.-Biomed. Tech., 60, 4, 2015, 301-316.

[6] Lucas P., David C., Ogheard F. et Bissig H., "EURAMET project 1291 - Comparison of primary standards for liquid micro flow rates", avril 2015, http://www. euramet.org/Media/docs/projects/EURAMET-P1291_ FLOW_Final_Report.pdf.

[7] Melvad C., Kruhne U. et FrederiKesen J., "Design considerations and initial validation of a liquid microflow calibration setup using parallel operated syringe pumps", Meas. Sci. Technol., 21, 7, 2010, 074004.

[8] IEC 60601-2-24, "Medical electrical equipment - Part 224: Particular requirements for the safety of infusion pumps and controllers", 1998. 